\title{
Microscale Pumping with Traversing Bubbles in Microchannels
}

\author{
Thomas K. Jun and Chang-Jin Kim \\ Mechanical and Aerospace Engineering Department \\ University of Califomia, Los Angeles \\ Los Angeles, CA 90095
}

\begin{abstract}
Pumping in microchannels using asymmetric heating and a single or multiple traversing bubble(s) is studied and demonstrated. This pumping mechanism requires no mechanical moving parts for actuation. A heat and mass transfer analysis was performed to understand the pumping mechanism and revealed that the two dominant forces are vapor pressure and surface tension. A pumping device with a channel hydraulic diameter of $3.4 \mu \mathrm{m}$ was fabricated on a silicon wafer using surface micromachining and tested. Testing results showed pumping velocities as high as $160 \mu \mathrm{m} / \mathrm{s}(505 \mathrm{pl} / \mathrm{min})$ with a pressure gain of approximately $800 \mathrm{~Pa}$.
\end{abstract}

\section{INTRODUCTION}

The effect of phase change in a fluid was studied to determine if this process could be used to achieve net motion of a fluid in a microchannel. A heat and mass transfer analysis revealed that the two dominant forces would be variations in vapor pressure and surface tension due to heater induced temperature gradient.

In this study, we introduce two methods to achieve net fluid movement in microchannels. The first method (Fig.la) consists of heating a single bubble and growing it in one direction, similar to the method used by Ozakis [1] in much larger metal channels ( $180 \mu \mathrm{m}$ vs. our $4 \mu \mathrm{m}$ hydraulic diameter). The second method (Fig.lb) utilizes multiple bubbles that are generated in sequence from left to right. The existing bubble(s) acts as an anchor during the subsequent nucleation, causing asymmetric liquid flow. The end result for both methods is net fluid displacement in the microchannel. (i)

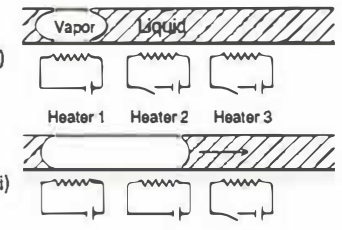

(iii)

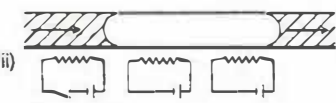

(a) Single bubble

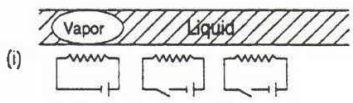

(ii)

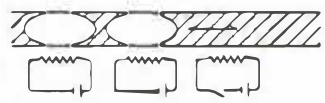

(iii)

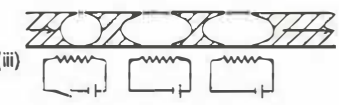

(b) Multiple bubbles
Figure 1. Two different modes of obtaining net fluid displacement

To verify the pumping principle, a test device was surface microachined as shown in Fig. 2. A series of heaters (six in the picture) are placed across the channel top shell. To allow for visual observation, we chose oxide for the shell layer and polysilicon as the sacrificial layer, unlike typical surface micromachining processes.

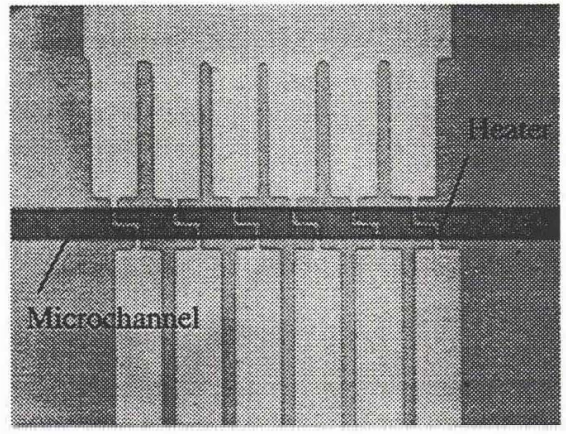

Figure 2. Optical photo of micropump and channel (channel has a hydraulic diameter of $\sim 4 \mu \mathrm{m}$ )

\section{THEORY}

A vapor bubble under asymmetric heating was modeled to understand the nature of the various forces that would be present (Fig. 1(a) case). The relevant forces are gradients in vapor pressure, surface tension (Marangoni forces) and momentum due to evaporation and condensation. The other method of obtaining net pressure in a duct (Fig. 1(b) case) is not analyzed in this section but is tested experimentally. By growing and collapsing a stationary vapor bubble, it could serve as an obstruction against flow in a channel and therefore function as a valve.

The vapor pressure of a liquid can get very large as the temperature of the liquid approaches the critical temperature. Heating a vapor bubble from one end should create a temperature gradient across the bubble. This temperature gradient would imply the presence of a net pressure difference across the end of the vapor bubble since vapor pressure is a strong function of temperature. A simple conduction model would predict a significant temperature difference between the heated and unheated end. However, due to the process of evaporation and condensation, a conduction only model is inadequate for this configuration. Therefore, a conjugate heat transfer model incorporating both heat and mass transfer was used.

The simplified geometry of the bubble and duct are shown in Fig. 3.

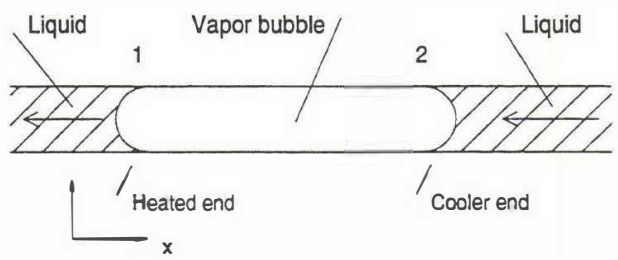

Figure 3. Geometry modeled by heat and mass transfer analysis 
The energy equation was applied across the vapor bubble in the duct

$$
\rho c \frac{\partial T}{\partial t}+u_{b} \frac{\partial(\rho c T)}{\partial x}=\frac{\partial}{\partial x}-\left(k \frac{\partial T}{\partial x}\right)+\frac{U P}{A_{c}} \cdot\left(T-T_{\infty}\right)
$$

where

$u_{b}=$ average bulk velocity in $\mathrm{x}$ direction

$p=$ density of fluid

$c=$ specific heat

$k=$ thermal conductivity of fluid

$T=$ temperature of fluid

$T_{-}=$ambient temperature

$\vec{U}=$ overall heat ransfer coefficient

$P=$ perimeter of duct

$A_{c}=$ cross sectional area of duct

The justification for eliminating the $y$ and $z$ terms of the energy equation is based on the assumption that the small cross sectional area of the microchannel is at approximately the same temperature. Similar to 1-D fin analysis, heat lost at the surface is accounted for by using a surface boundary condition, which is the last term in Eq. (1). Next the momentum equation is applied to flow in a duct as

$$
u \frac{\partial(p u)}{\partial x}=-\frac{\partial p}{\partial x}+\mu \frac{\partial}{\partial y}\left[\frac{\partial u}{\partial y}\right]
$$

where

$u=$ velocity in $\mathrm{x}$ direction

$p=$ pressure in fluid

$\mu=$ fluid viscosity

The well known Darcy friction factor solution is

$$
f=\frac{(-d p / d x) D_{H}}{(1 / 2) \rho u_{b}^{2}}=\frac{K}{\operatorname{Re}_{D}} ; \frac{d p}{d x}=\frac{\Delta p}{L}
$$

where

$\mathrm{Re}=$ Reynolds number

$K=$ friction constant ( ranges from 64 to 90 )

$D_{H}=$ hydraulic diameter

$\boldsymbol{L}=$ length of vapor bubble

The constant $\mathrm{K}$ ranges from 64 for a macro scale circular duct to about 80 for microscale ducts as reported by Pfahler et al [2]. From Eq.(2) the pressure gradient in the duct must be linear at steady state. From kinetic theory of gas the evaporation at the hot interface is approximated as

$$
\dot{m}^{\prime \prime}=\left(p_{s a t}-p_{b u b b l e}\right) \sqrt{\frac{1}{2 \pi R T_{s a t}}}
$$

where

$p_{\text {sat }}=$ saturation pressure at interface

$p_{\text {bubble }}=$ pressure in bubble

$R=$ gas constant

$T_{\text {sat }}=$ saturation temperature

Next the interfacial conditions must be satisfied at the vapor-liquid interface. Using an energy and mass balance at the vapor-liquid interface

$$
\dot{m}_{1}^{\prime \prime} h_{f s}=-k\left[\frac{d T}{d x}\right]_{l_{1}}+k\left[\frac{d T}{d x}\right]_{v_{1}}
$$

at interface 1 (hot end), and

$$
-\dot{m}_{2}^{\prime \prime} h_{f_{g}}=-k\left[\frac{d T}{d x}\right]_{v_{2}}+k\left[\frac{d T}{d x}\right]_{l_{2}}
$$

at interface 2 (cold end). Furthermore

$$
\begin{aligned}
\dot{m}_{1}^{\prime \prime}=\dot{m}_{2}^{\prime \prime} & =\left(\rho_{v} v_{v}\right)_{1}=\left(\rho_{l} v_{l}\right)_{1} \\
& =\left(\rho_{v} v_{v}\right)_{2}=\left(\rho_{l} v_{l}\right)_{2}
\end{aligned}
$$

$\dot{m}_{1}^{\prime \prime}=$ mass flux at interface 1 (hot end)

$\dot{m}_{2}^{\prime \prime}=$ mass flux at interface 2 (cold end)

The temperature boundary and initial conditions are defined as

$$
\begin{aligned}
& T(x \rightarrow \infty)=T_{\infty} \quad ; T(t=0)=T_{0} \\
& T(x=0)=T_{\text {heater }} \text { or }-k \frac{d T}{d x}=q_{\text {heater }} \\
& p_{1}(\mathrm{x}=0)=p_{\text {sat }}\left(T_{1}\right) ; p_{2}(x=L)=p_{\text {sat }}\left(T_{2}\right)
\end{aligned}
$$

Solving Eqs. (1) and (4) simultaneously along with the interfacial and boundary conditions, the temperature, evaporation rate and pressure were found for various heater configuration and vapor bubble lengths. It was found that the pressure difference increased with bubble length as expected.

As stated earlier, there are other factors that may cause a net pressure difference in the duct. Surface tension is a fairly strong function of temperature, hence in the presence of a substantial temperature gradient, the two ends of the vapor bubble can have an interfacial pressure difference known as the Marangoni force [3]. The Marangoni force was approximated as

$$
F=\frac{2}{R} \Delta \sigma A
$$

$R=$ radius of curvature of vapor bubble

$\Delta \sigma=$ difference of surface tension coefficient across vapor bubble $A=$ cross sectional area of vapor bubble

The final force that was calculated was the vapor recoil force that was produced due to the evaporation and condensation process and can be high if the evaporation rate is very large. Applying the Reynolds transport theorem for momentum at the liquid and vapor interface, the net force due to evaporation and condensation is

$$
F_{x}=\left(\rho V^{2} A\right)_{\text {vapor }}-\left(\rho V^{2} A\right)_{\text {liquid }}
$$

The effect of condensation is similar to the evaporation process, only now the fluid experiences a deceleration, and so can be modeled in the same way. Using Eq.(12) and typical evaporation rates, the pressure change due to evaporation or condensation was found to be very small $(<1 \mathrm{~Pa})$. As a result, for this analysis, pressure changes due to evaporation or condensation was assumed to be negligible. 


\section{FABRICATION}

To verify the principles analyzed in the previous section a surface micromachined pumping device was fabricated as shown in Fig. 4. The fabrication process started with the deposition of $2.0 \mu \mathrm{m}$ of low temperature oxide (LTO) and $2.0 \mu \mathrm{m}$ of polysilicon using LPCVD. Then the sacrificial polysilicon layer is doped with spin-on phosphorus dopant to make the layer $\mathrm{n}^{+}$. This $\mathrm{n}^{++}$ property will later enhance the dry etching rate in $\mathrm{XeF}_{2}$. This polysilicon layer is then pattemed using RIE to form the sacrificial layer that will later become the void space in the microchannel. Next $2.0 \mu \mathrm{m}$ of LTO is deposited to form the transparent shell of the microchannel (Fig. 4a). Then $0.7 \mu \mathrm{m}$ of polysilicon is deposited and patterned using RIE to form the heater that lies on top of the microchannel shell (Fig. 4b). Using buffered oxide etchant (BOE), holes are pattemed into the oxide layer to expose the sacrificial polysilicon at the ends of the microchannel (Fig. 4c). With photoresist covering the entire wafer except for the oxide holes, the dry etchant $\mathrm{XeF}_{2}$ is used to etch the sacrificial polysilicon core of the microchannel. The finished devices consist of a hollow oxide shell with polysilicon heaters running along the top surface of the oxide shell as illustrated in Fig. 4 d.

(a)
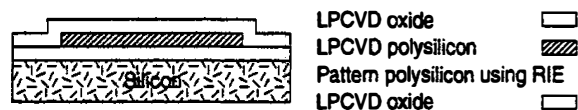

(b)

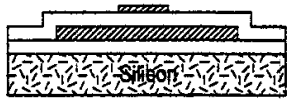

LPCVD polysilicon Dope poly using SOG dopant Pattern polysilicon heater

(c)

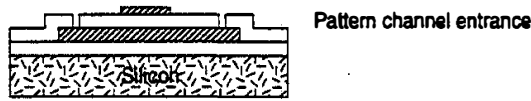

(d)

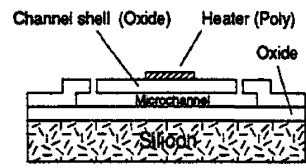

Figure 4. Process flow

\section{TESTING}

The micropump was tested to verify the physical principles as described in the theory section. The important pump parameters that were investigated consisted of pumping pressure head, flow velocity and Reynolds number.

The testing was done by powering the heaters in sequence using a pulse signal pattern similar to that shown in Fig. 5. Sample test cases of isopropanol pumping in a $726 \mu \mathrm{m}$-long, 29 $\mu \mathrm{m}$-wide, $2 \mu \mathrm{m}$-high microchannel are presented. The three parameters studied were heater pulse amplitude (voltage), pulse width and pulse overlap as shown in Fig. 5. The heater pulse amplitude is simply the heater input voltage and so is directly related to the output power of the line heater. The heater pulse width is the time period that the heater is powered and the pulse percent overlap is defined as the overlap of the power input for adjacent heaters. In this study, these parameters were varied to understand their effect on net fluid flow in a microchannel.

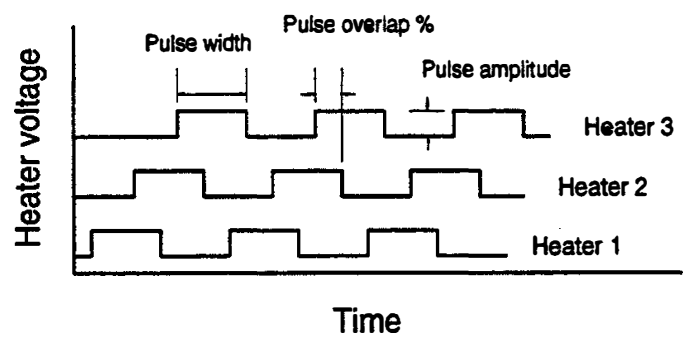

Figure 5. Heater input signal and important parameters

As stated previously, two methods were used to achieve net fluid movement as illustrated in Fig. 1. The first method consisted of heating a single bubble and causing translation of the interface due to the forces mentioned. The second method utilizes multiple bubbles that are generated in sequence from left to right. The existing bubble(s) acts as an anchor during the subsequent nucleation, causing asymmetric liquid flow. The end result of both methods is net fluid displacement in the microchannel.

In this study, three heaters were used to produce net fluid flow in a $3.4 \mu \mathrm{m}$ hydraulic diameter microchannel. The heater pulse amplitude was varied from 18 to 26 Volts and the pulse width ranged from 0.25 to 1 second. In addition, the pulse overlap ranged from 50 to 90 percent overlap. The three heater signals were produced using a function generator that was controlled using a personal computer. In tum the function generator produced a series of synchronized square pulses with the desired pulse amplitude, width and overlap for each heater as shown in Fig. 5. The testing of the micropump required no special priming signal to initially start the pumping and allowed the same steady state signals to be used from start to finish.

\section{RESULTS AND DISCUSSION}

\section{Numerical results of relevant forces}

The heat and mass transfer analysis revealed that the two significant forces were variation in vapor pressure and surface tension caused by the heater induced temperature gradients. It was found that at high temperatures, when evaporation rates were high, the vapor pressure forces dominated due to the significant vapor pressure present. However, when the evaporation rates dropped and the temperature between the hot and the cold end got larger, the surface tension became more significant [3]. It was also found that the forces due to vapor recoil were very small for even the largest possible evaporation rates. Table. 1 shows the relative magnitude of the calculated forces.

\section{Net Pressure}

$\begin{array}{lr}\text { Vapor Pressure } & 600 \mathrm{~Pa} \\ \text { Marangoni Pressure } & 10 \mathrm{~Pa} \\ \text { Vapor Recoil } & <0.5 \mathrm{~Pa}\end{array}$

Table 1. Comparison of calculated forces present in microchannel with $130 \mu \mathrm{m}$ long vapor bubble (three heater lengths) at $\sim 92{ }^{\circ} \mathrm{C}$ with $\Delta T=0.1^{\circ} \mathrm{C}$.

\section{Experimental Results of micropumping device}

Fig. 5 illustrates the basic operational envelope of the micropump operating at a heater voltage of $24 \mathrm{~V}$. This voltage 
translates to $58 \mathrm{~mW}$ to each heater, which was found to be the minimum power required to obtain reliable operation of the micropump. The failure mode at power inputs lower than $55 \mathrm{~mW}$ (23 Volts) was usually due to failed bubble nucleation at one or more heater sites.

As Fig. 6 shows, the lower and upper limits of the pulse percent overlap was found to be approximately 60 to 85 percent pulse overlap. At pulse overlap greater than about $85 \%$ the failure mode was found to be little net displacement of fluid due to the three bubbles nucleating at nearly the same time. The end result was equal displacement of fluid to both sides of the microchannel. This is due to the finite time required for bubble nucleation and growth. Without enough time lag between adjacent heaters, the sequential bubble generation from left to right was lost. To investigate this phenomenon, the input power to the heater was increased in an attempt to shorten the time for bubble generation and growth, thereby getting rapid but sequential bubble generation at the three heaters. It was found, however, that in this case one large symmetrical bubble would nucleate and prevent the pump from operating. At the lower pulse overlap limit, the failure mode was usually due to the premature collapse of the previously generated bubble(s). In the case where the pump was operating in the multiple bubble mode (Fig. 1b) this caused the pump to lose the anchor effect of the earlier bubbles and so operation was less than ideal. For the single bubble operation, premature collapse meant that the single bubble started collapsing on the starting side and thus lost the increased net pressure effect of having a longer bubble.

Testing also revealed that heater power input and pulse width were the parameters that determined whether the pump would operate in single or multiple bubble mode. Heater power, however, was found to be the more significant deciding parameter of the two. When the heater was powered at a high voltage of $26 \mathrm{~V}$ (62 mW/heater), the pump operated almost exclusively in the single asymmetrically growing bubble mode when testing within the operational window described earlier. However when the pump was operated at a lower voltage of $23 \mathrm{~V}(55 \mathrm{~mW} / \mathrm{heater})$ the pump always operated in the multiple bubble mode.

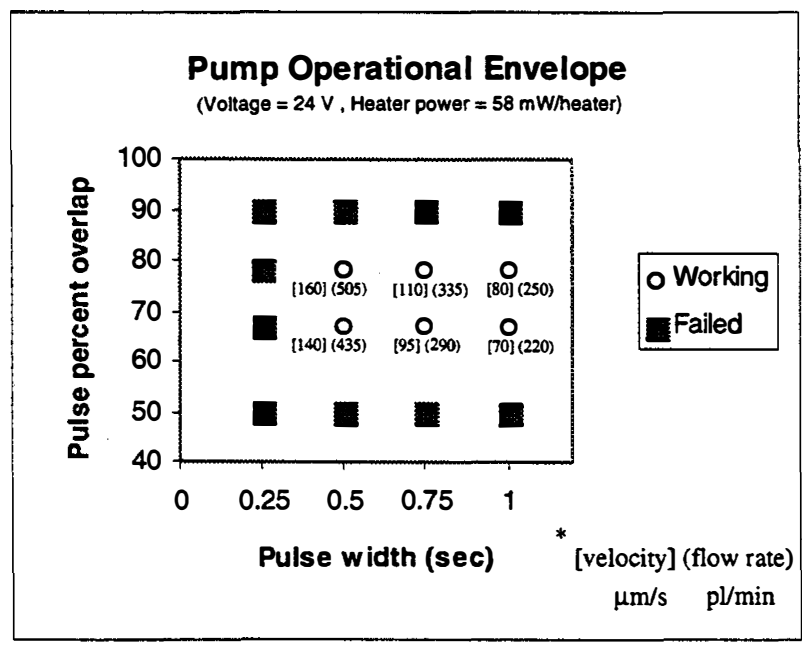

Figure 6. Operation envelope of the micropump

The pulse width also influenced the operational mode of the pump. Testing at $24 \mathrm{~V}$ and $67 \%$ overlap, the pump operated in the multiple bubble mode for pulse width of 0.5 seconds but changed to the single bubble mode when the pulse width was increased to $1 \mathrm{~s}$. Surprisingly pulse overlap had less of an effect on operational mode determination. Intuitively one would guess that larger pulse overlap would result in single bubble mode of operation but this was not found to be the case at least within the operational window tested.

During testing there was no observable liquid microlayer between the vapor bubbles and the channel walls, which suggests there would be little liquid slipping past the bubble, if any. It was indeed visually confirmed that the motion of the actuated vapor bubble at the center was duplicated by the vapor bubbles away from the heated zone, leading us to conclude that the ranslating vapor bubble in fact causes net motion of the column of fluid. The above was confirmed when we succeeded in pushing vapor bubbles out of one end of the microchannel. This direct displacement also allowed us to obtain the flow rate from the bubble motion. Assuming that the pump was operating in quasisteady state, the flow rate and the solution for steady laminar flow in a duct was used to estimate the pressure supplied by the pump.

\section{CONCLUSIONS}

The feasibility of pumping fluids in a $3.4 \mu \mathrm{m}$ hydraulic diameter microchannel using asymmetric heating and traversing bubbles has been demonstrated. A heat and mass transfer analysis has been done to estimate the magnitude of the relevant forces present. The analysis revealed that variations in vapor pressure and surface tension (Marangoni effect) are the two dominant forces present.

The advantage of this present design is the complete absence of any moving mechanical parts or small component assembly. Furthermore the proposed design can be integrated in standard silicon processing..

Test results has shown that our early generation design is already capable of delivering velocities as high as $160 \mu \mathrm{m} / \mathrm{s}$ in a $3.4 \mu \mathrm{m}$ hydraulic diameter, $726 \mu \mathrm{m}$ long microchannel. This corresponds to pressure heads as large as $800 \mathrm{~Pa}$, and a flow rate of $0.5 \mathrm{nl} / \mathrm{min}$. With optimization of both pump design and operation significant improvements is expected in the performance of future versions.

\section{ACKNOWLEDGMENT}

The authors wish to thank J. Simon for his numerous assistance in processing and Prof. C.-M. Ho and Dr. K. Schadow for various discussions. This work is in part supported by the Office of Naval Research under the Grant N00014-94-1-0536

\section{REFERENCES}

1. Koichi Ozaki, "Pumping Mechanism Using Periodic Change of a Fluid", Proc. IEEE Micro Electro Mechanical Systems, Amsterdam, Netherlands, Jan.-Feb. 1995, pp.31-36.

2. J. Pfahler, J. Harley and H. Bau, "Gas and Liquid Flow in Small Channels",ASME Proc HTD-Vol.32, Micromechanical Sensors, Actuators, and Systems, 1991, pp.49-60.

3. J. L. McGrew, T. L. Rehm and R. G. Griskey, "The Effect of Temperature Induced Surface Tension Gradients on Bubble Mechanics", Appl. Sci. Res., June 1974, pp.195-210.

4. M. Fahndrich, B. Hochwind and A. Zollner, "Fluid Dynamics in Micro Dosing Actuators", The $8^{\text {th }}$ International Conference of Solid-State Sensors and Actuators, (Transducers '95), Stockholm, Sweden, June 1995, pp.295-298.

5. A. F. Mills, "Heat and Mass Transfer", Richard D. Irwin, Inc., Homewood, IL, 1995.

6. V. S. Arpaci, "Conduction Heat Transfer", Addison-Wesley, Reading, MA, 1966 